\title{
STATES ON THE CUNTZ ALGEBRAS AND $p$-ADIC RANDOM WALKS
}

\author{
P. E. T. JORGENSEN and A. M. PAOLUCCI ${ }^{\bowtie}$ \\ (Received 2 August 2010; accepted 16 December 2010) \\ Communicated by M. Varghese \\ Dedicated to Alan Carey, on the occasion of his 60th birthday
}

\begin{abstract}
We study Markov measures and $p$-adic random walks with the use of states on the Cuntz algebras $O_{p}$. Via the Gelfand-Naimark-Segal construction, these come from families of representations of $O_{p}$. We prove that these representations reflect selfsimilarity especially well. In this paper, we consider a Cuntz-Krieger type algebra where the adjacency matrix depends on a parameter $q(q=1$ is the case of Cuntz-Krieger algebra). This is an ongoing work generalizing a construction of certain measures associated to random walks on graphs.

2010 Mathematics subject classification: primary 47B47; secondary 81P15, 60G35, 33D50, 46C05, 46L52, 11D88, 33D80.

Keywords and phrases: commutator, quantum theory, signal processing, p-adic analysis, Hilbert space, spectrum.
\end{abstract}

\section{Introduction}

In this paper, we study a family of $q$-deformations and their representations. The base point for our analysis is a family of representations of the Cuntz algebras. The fundamental principle underlying our approach is the use of iterations in subdivisions of systems of orthogonal projections. This allows us to make precise notions of selfsimilarity and renormalization. Quite a number of ideas are involved and, for the reader's convenience, they are outlined below in the introduction. Thus, a main part of our paper serves to present the wider framework: this is all leading up to Theorem 5.3 in Section 5.

We will treat a family of measures whose construction is a natural outgrowth of the study of the representations of $C^{*}$-algebras on generators and relations. In this paper, we will detail the connection between these measures and families of representations of purely infinite $C^{*}$-algebras.

The first author (PJ) thanks the US NSF for partial support.

(C) 2011 Australian Mathematical Publishing Association Inc. 1446-7887/2011 \$16.00 
The $C^{*}$-algebras we have in mind will include the Cuntz algebras, and the Cuntz-Krieger algebras and their $q$-deformations. But, rather than the $C^{*}$-algebras themselves, our focus will be on their representations. Now it is known since Glimm [13] that there are purely infinite $C^{*}$-algebras whose representations do not have Borel cross sections in the sense of Mackey. The Cuntz algebras fall in this singular class. More specifically, the equivalence classes of irreducible representations of $O_{N}, N>1$, cannot be parameterized by a Borel cross section. Intuitively, there are too many representations: it is a theorem that one is not able to produce a classification that is Borel measurably indexed.

Nonetheless, in connection with such applications of representation theory as the study of wavelets, Markov measures, and $q$-families of Riemann zeta functions (see details and cited references below), it has emerged that there are infinite families of irreducible representations of $O_{N}, N>1$, and of related $C^{*}$-algebras which unexpectedly accomplish such tasks as classification of wavelet families, producing a tool for $p$-infinite product factorization of $q$-Riemann zeta functions, and producing Markov measures.

The representations reflect an underlying $N$-adic subdivision scheme. And, the Cuntz algebra $O_{N}$ is precisely reflecting the same kind of $N$-adic subdivision; see details below. Hence, by passing to the natural maximal abelian subalgebra $M_{N}$ in $O_{N}$, we get measures by restricting the states on $O_{N}$. Indeed, the maximal abelian subalgebra $M_{N}$ is of the form $C\left(K_{N}\right)$, where $K_{N}$ is the Cantor set corresponding to $N$-adic subdivision and $C\left(K_{N}\right)$ is the $C^{*}$-algebra of all continuous functions on $K_{N}$. In a number of recent papers, the present coauthors have considered the measures on $K_{N}$ which arise from restriction states on $O_{N}$, with special attention to applications to Markov measures, and the study of $q$-families of Riemann zeta functions, producing new $p$-infinite product factorization of $q$-Riemann zeta functions, and for producing Markov measures, quantum deformations, representations of $p$-adic groups, graph algebras, and the Bernoulli numbers over $p$-adic number fields. The outline of the paper is as follows: in Section 2, we give the fundamentals for the construction of projection-valued measures for the Cuntz algebra and Cuntz-Krieger algebra used in Sections 3 and 5, respectively. In this paper, we aim at studying a Cuntz-Krieger type algebra where the adjacency matrix depends on a parameter $q(q=1$ is the case of Cuntz-Krieger algebra). In Section 3, we look at the $p$-adic representations of the Cuntz algebra $O_{p}$ related to wavelets. These representations will be used in Section 5 to construct certain measures associated to random walks on graphs via Perron-Frobenius eigenvectors.

1.1. Discussion of the literature. Our study of representations of the Cuntz relations [5, 6] is closely tied with the study of endomorphisms of $B(H)$, where $H$ is a separable (infinitely dimensional) complex Hilbert space. For treatments of this in the literature, we refer to the paper [8] and the references cited there; and there are connections of our present results to wavelets [1, 2, 4, 7, 15, 16, 18]. For the theory of $q$-deformations and its varied applications, see, for example, [20, 22, 23]. 
For general background on Fourier analysis and special functions, relevant to the present discussion, see [12]; noncommutative geometry [27]; graph algebras [30]; number theory and zeta functions [14]; representation theory [11, 17, 21, 24]. Relevant references to the theory of invariant measures are [9, 10, 15, 19].

\section{Projection-valued measures}

We begin with some comments to link the ideas from the introduction with the main theme of our paper.

Our understanding of a number of problems in mathematical physics (for example, in quantum information) is facilitated by the introduction of a Cuntz algebra, or a family of Cuntz-Krieger algebras; see the papers cited above. The list of these problems includes a variety of occurrences of selfsimilarity, rescaling, or renormalization (for example, by affine mappings, by conformal mappings, or by Markov processes). Via associated selfsimilar measures $\mu$ (for example, maximalentropy measures, Markov measures, and so on) this approach to problems in turn suggests new and useful symbolic encodings; encoding the problem at hand into a suitable Hilbert space $H:=L^{2}(\mu)$ representing the states in a physical system.

Further, the $C^{*}$-algebras and their representations help us to link questions in dynamics with symbolic models. A key feature is that the Cuntz or Cuntz-Krieger algebras are generated by a finite number of partial isometries, subject to relations. (While there are models with an infinite number of generators, here we restrict to a finite number; for example, $O_{N}$ for $N$ finite. In the study of subband filters and associated wavelets, $N=$ the number of frequency subbands.)

By definition, the generating partial isometries have orthogonal ranges adding up to the whole Hilbert space $H$. Hence, when we use finite words formed from the generators, we arrive at arbitrarily fine subdivisions of the Hilbert space $H$ into orthogonal blocks, and hence of the states of the system under consideration. Thus, the model yields similarity of scales: patterns in large scale are repeated in arbitrarily small scales. Moreover, such orthogonal splittings are used for example in error correction codes from quantum information theory.

Our aim in this paper is to turn around this process: starting with a Cuntz or CuntzKrieger algebra, we will use symbolic dynamics in a construction of the measures $\mu$ which enter into the representations, and offer the corresponding Hilbert space $L^{2}(\mu)$ as a repository for physical states. This is especially successful in the study of (oneparameter) quantum deformations; that is, $q$-deformations, where of course, for $q=1$ one gets back the case of Cuntz-Krieger algebras considered in the work by one of the authors with Marcolli [29]. Our discussion here stresses the review and an outline of new interdisciplinary connections.

We note (Section 3) that our methods also yield certain intriguing $p$-adic representations of the Cuntz or Cuntz-Krieger algebras. The applications here include: Markov measures, $p$-adic random walks, $q$-zeta functions, and infinite Bernoulli convolutions. 
The main idea below relies on a basic property of the Cuntz algebra and its representations. We recall that one denotes by $O_{N}$ the $C^{*}$-algebra generated by $N$, $N \in \mathbf{N}$, isometries $S_{0}, \ldots, S_{N-1}$ satisfying

$$
S_{i}^{*} S_{j}=\delta_{i j} \mathbf{1}
$$

and

$$
\sum_{i=0}^{N-1} S_{i} S_{i}^{*}=\mathbf{1},
$$

where $i, j=0, \ldots, N-1$. Since each Cuntz algebra $O_{N}$ contains a canonical abelian subalgebra $C\left(K_{N}\right)$ with $N$-scaling, and a Cantor set $K_{N}$, it is natural to look for states of the abelian $C^{\star}$-algebra $C\left(K_{N}\right)$. By the Riesz representation theorem, states on $C\left(K_{N}\right)$ are in bijective correspondence with measures on $K_{N}$; and they are often identified. These are outlined below. We construct certain projection-valued measures by studying subdivisions of compact metric spaces and subdivisions of projections in Hilbert spaces. We apply this construction first to the case of the Cuntz algebra $O_{N}$ extending in Section 5 to a more general type of Cuntz-Krieger algebra (see [29] for the case of the Cuntz-Krieger algebra). Note that here we apply the construction to the case of $O_{N}$. This will be used in Section 3 to construct a measure associated to wavelet representations of $O_{N}$.

2.1. Gelfand space. In the study of Cuntz (or $\mathrm{C}-\mathrm{K}$ ) algebras, certain natural abelian subalgebras enter as follows. As we show, they are essential in our understanding of, for example, decomposition theory for representations. The particular, Cuntz (or $\mathrm{C}-\mathrm{K}$ ) algebra itself arises as a $C^{\star}$-norm closure of the span of certain finite words in specific generators. As follows from our application presented here, the abelian subalgebras arise in the same way (from encoded words), but now with commuting families of only special finite words in the generators. The Gelfand-Naimark theorem applies to every one of these abelian subalgebras, and so offers representations of each by a model $C^{\star}$-algebra $C\left(K_{N}\right)$ with $K_{N}$ here denoting the Gelfand space. Of course, a priori, a Gelfand space is only determined up to homeomorphism in the category of compact Hausdorff spaces. As a result, care must be exercised: the conclusion from the Gelfand-Naimark theorem alone only yields information up to $C^{\star}$-isomorphism. Such a $C^{\star}$-isomorphism offers a representation of a particular abelian subalgebra. A different abelian subalgebra will have a different representation of $C\left(K_{N}\right)$. As we show here, to understand the role of these representations, more work must be done: our aim here is to call attention to this, and to get a concrete understanding and realization of $K_{N}$. We show that indeed $K_{N}$ may be realized in a fairly concrete manner as a Cantor space. The concreteness of such representations is important in applications, for example to error-correction codes $[25,26]$. For this part, we also refer to the cited earlier papers.

Definition 2.1. Let $(X, d)$ be a compact metric space. For subsets $A \subset X$, define the diameter

$$
|A|:=\sup \{d(x, y) \mid x, y \in A\}
$$


A partition of $X$ is a family $\{A(i)\}_{i \in I}$, where $I$ is some index set, such that

$$
\bigcup_{i} A(i)=X
$$

and

$$
A(i) \cap A(j)=\emptyset \quad \text { if } i \neq j .
$$

Let $N \in \mathbf{Z}_{+}, N \geq 2$, and $\Gamma_{N}:=\{1, \ldots, N\}$. Suppose that for each $k \in \mathbf{Z}_{+}$we have a partition into Borel subsets $A_{k}(a)$ indexed by $a \in \Gamma_{N}^{k}=\Gamma_{N} \times \cdots \times \Gamma_{N} k$ times and $|A|=0\left(N^{-c k}\right), c>0$. If every $A_{k+1}(a)$ is contained in some $A_{k}(b)$, we say that $\left\{A_{k}(a)\right\}$ is an $N$-adic system of partitions of $X$.

Definition 2.2. Let $\mathcal{H}$ be a complex Hilbert space. A partition of projections in $\mathcal{H}$ is a system $\{P(i)\}_{i \in I}$ of projections, that is,

$$
P(i)=P(i)^{\star}=P(i)^{2}
$$

such that

$$
P(i) P(j)=0 \quad \text { (orthogonality) }
$$

if $i \neq j$, and

$$
\sum P(i)=1_{\mathcal{H}} \quad \text { (completeness), }
$$

where $1_{\mathcal{H}}$ is the identity operator in $\mathcal{H}$. Suppose that for every $k \in \mathbf{Z}_{+}$we have projections $\{P(a)\}_{a \in \Gamma_{N}^{k}}$ such that every $P(a)_{k+1}$ is contained in some $P(b)_{k}$, that is, $P(b)_{k} P(a)_{k+1}=P(a)_{k+1}^{N}$; then the combined system $\left\{P(a)_{k}\right\}_{k \in \mathbf{Z}_{+}, a \in \Gamma_{N}^{k}}$ is a system of partitions of $1_{\mathcal{H}}$ forming, by $N$-adic subdivisions, an $N$-adic system of projections. We call such a system an $N$-adic system of partitions of $1_{\mathcal{H}}$ into projections.

Definition 2.3. Denote by $B(X)$ the collection of all Borel subsets of the compact metric space $X$. A positive operator-valued function $E$ defined on $B(X)$ is called a $\sigma$-additive measure if, given a sequence $B_{1}, B_{2} \ldots$ in $B(X)$ such that $B_{i} \cap B_{j}=\emptyset, i \neq j$, then

$$
E\left(\bigcup_{i} B_{i}\right)=\sum_{i} E\left(B_{i}\right) .
$$

Note that the values $E\left(B_{i}\right)$ are positive operators (or nonnegative, that is, $\langle\xi, E(B) \xi\rangle \geq 0$ ), so we may take the summation on the right-hand side to be convergent in the strong operator topology.

Definition 2.4. A $\sigma$-additive function $E: B(X) \rightarrow$ (positive operators) is called a positive operator measure. We say that a positive operator measure $E$ is an orthogonal projection-valued measure if one of the two equivalent conditions (a) and (b) is satisfied.

(a) $E(A \cap B)=E(A) E(B)$, for all $A, B \in B(X)$.

(b) $E(A) E(B)=0$ if $A, B \in B(X)$ and $A \cap B=\emptyset$. 
Recall that we have the following lemma.

Lemma 2.5. Let $N \in \mathbf{Z}_{+}, N \geq 2$. Let $(X, d)$ be a compact metric space and let $\mathcal{H}$ be a complex Hilbert space. Let $\left\{A_{k}(a)\right\}_{k \in \mathbf{Z}_{+}, a \in \Gamma_{N}^{k}}$ be an $N$-adic system of partitions of $X$ and let $\left\{P_{k}(a)\right\}_{k \in \mathbf{Z}_{+}, a \in \Gamma_{N}^{k}}$ be the corresponding $N$-adic system of projections. Then there is a unique normalized orthogonal projection-valued measure E(·) defined on the Borel subsets of $X$ and taking values in the orthogonal projections in $\mathcal{H}$ such that

$$
E\left(A_{k}(a)\right)=P_{k}(a)
$$

for every $k \in \mathbf{Z}_{+}, a \in \Gamma_{N}^{k}$.

Proof. See [15].

Let $S_{0}, \ldots, S_{N-1}$ be the generators of $O_{N}$ and let the operators $P_{k}(a)$ be defined by

$$
P_{k}(a):=S_{a_{1}} S_{a_{2}} \cdots S_{a_{k}} S_{a_{k}}^{\star} \cdots S_{a_{1}}^{\star},
$$

where $a=\left(a_{1}, a_{2}, \ldots, a_{k}\right) \in \Lambda_{A}^{k}$ are total sets in the abelian algebra $C\left(\Lambda_{A}\right)$. For every $k \in \mathbf{Z}_{+}$, the finite sums $\sum_{a \in \Lambda_{A}^{k}} c_{a} \chi_{A_{k}(a)}$ form an algebra of functions $U_{k}$ on $X$ and, from the definition of the partition system $\left\{A_{k}(a)\right\}_{k \in \mathbf{Z}_{+}, a \in \Gamma_{N}^{k}}$, it follows that there are natural embeddings $U_{k} \rightarrow U_{k+1}$. Thus, the mapping defined for every $k \in \mathbf{Z}_{+}$by

$$
\sum_{a \in \Gamma_{N}^{k}} c_{a} \chi_{A_{k}(a)} \rightarrow \sum_{a \in \Gamma_{N}^{k}} c_{a} P_{k}(a)
$$

extends to the algebra $U:=\bigcup_{k \in \mathbf{Z}_{+}} U_{k}$. The algebra $U$ is closed under conjugation and the $\star$. Denote by $\pi$ the mapping defined above. By the Definitions 2.1, 2.2 and 2.4 we have that $\pi\left(f_{1} f_{2}\right)=\pi\left(f_{1}\right) \pi\left(f_{2}\right)$ with $f_{1}, f_{2} \in U$ and $\pi(\bar{f})=\pi(f)^{\star}$, with $f \in U$. We observe that any function $f \in C(X)$ may be uniformly approximated with sequences in $U$ since the sets $A_{k}(a)$ satisfy $|A|=0\left(N^{-c k}\right), c>0$. Then the map $\pi$ by a standard argument from function theory extends from $C(X)$ to all the Baire functions and the extension preserves products and adjoints. Call such an extension $\tilde{\pi}$. Define a projection-valued measure by the following formula:

$$
E(B):=\widetilde{\pi}\left(\chi_{B}\right),
$$

where $\chi_{B}$ is the indicator function of the set $B$. The measure $\tilde{\pi}$ is obtained as a unique extension from the measure $\pi$, so it follows that $E(\cdot)$ satisfies the properties of definition 2 and it is countably additive. Also, it satisfies $E\left(A_{k}(a)\right)=P_{k}(a)$ for every $k \in \mathbf{Z}_{+}, a \in \Gamma_{N}^{k}$. This system of partitions will be used in the following sections to build measures out of representations of the Cuntz algebra. To do that, let us recall from [15] the following lemma.

Lemma 2.6. Let $N \in \mathbf{Z}_{+}, N \geq 2$, and let $S_{0}, S_{1}, \ldots, S_{N-1}$ be a representation of $O_{N}$ on a Hilbert space $\mathcal{H}$. For $k \in \mathbf{Z}_{+}$and $a=\left(a_{1}, a_{2}, \ldots, a_{k}\right) \in \Gamma_{N}^{k}$, set

$$
S_{a}:=S_{a_{1}} S_{a_{2}} \cdots S_{a_{k}}
$$

and $P_{k}(a)=S_{a} S_{a}^{\star}$. Then the system $\left\{P_{k}(a)\right\}$ is a system of partitions of $1_{\mathcal{H}}$ into projections. 
Proof. For convenience, we sketch some steps from the proof. The projections $P_{k}(a)$ generate an abelian subalgebra of operators. If two operators $S, T$ are positive on a Hilbert space $\mathcal{H}$, then we say that $S \leq T$ if $\langle x, S x\rangle \leq\langle x, T x\rangle$. Then

$$
\begin{aligned}
\sum_{i} P_{k+1}(a i) & =\sum_{i} S_{i_{1}} \cdots S_{i_{k}} S_{i} S_{i}^{\star} S_{i_{k}}^{\star} \cdots S_{i_{1}}^{\star} \\
& =S_{i_{1}} \cdots S_{i_{k}} \sum S_{i} S_{i}^{\star} S_{i_{k}}^{\star} \cdots S_{i_{1}}^{\star}=S_{i_{1}} \cdots S_{i_{k}} S_{i_{k}}^{\star} \cdots S_{i_{1}}^{\star}=P_{k}(a),
\end{aligned}
$$

using the defining relations of the Cuntz algebra. Thus, $P_{k+1}(a i)=P_{k}(a i)$, that is, $P_{k}(a) P_{k+1}(a i) \leq P_{k+1}(a)$.

We consider the induced measure given by the following formula:

$$
\mu_{f}(\cdot):=\langle f, E(\cdot) f\rangle=\|E(\cdot) f\|^{2},
$$

where $f \in \mathcal{H}$, referring to a projection-valued measure applied to the vector $f$.

If $\|f\|=1$ and $X=[0,1]$, then $\mu_{f}$ is a probability measure.

\section{Representations of the Cuntz algebras}

It turns out that the Cuntz algebra and its representations offer a natural framework for $p$-adic harmonic analysis. This is sketched below. We now turn to the correspondence between states and representations; and we discuss the $p$-adic representations.

We specialize this measure by means of $p$-adic representations and of $O_{p}$ and we use this construction later in Section 5. From now on, let us assume that $N=p$ is a prime in the definition of the Cuntz algebra.

We define a class of representations of the Cuntz algebra in the space of (complexvalued) functions on the $p$-adic disk. These representations are unitarily equivalent to the representations constructed by Bratteli and Jorgensen in [3]. Let $L^{2}\left(\mathbf{Z}_{p}\right)$ be the space of $L^{2}$-integrable functions on a $p$-adic disk. Define the characteristic function of a $p$-adic disk by

$$
\chi_{k}\left(x-x_{0}\right)=\chi\left(p^{k}\left|x-x_{0}\right|_{p}\right)
$$

such that $\chi(t)=0$ if $t \geq 1$ and $\chi(t)=1$ if $t \leq 1, x, x_{0} \in \mathbf{Z}_{p}$ being the ring of $p$-adic integers numbers. For the function, we have $\chi_{k}\left(x-x_{0}\right)=1$ on the disk $D\left(x_{0}, p^{-k}\right)$ of radius $p^{-k}$ with center in $x_{0}$ and $\chi_{k}\left(x-x_{0}\right)=0$ outside this disk. A representation of the Cuntz algebra in $L^{2}\left(\mathbf{Z}_{p}\right)$ has the following form for the generators $S_{i}$ and $S_{i}^{\star}$ :

$$
S_{i} \xi(x)=\sqrt{p} \chi_{1}(x-i) \xi\left(\left[\frac{1}{p} x\right]\right)
$$

and

$$
S_{i}^{\star} \xi(x)=\frac{1}{\sqrt{p}} \xi(i+p x),
$$


where $[x]=x-x \bmod 1$ for $x \in \mathbf{Q}_{p}$ is the integer part of $x$ and $\chi_{1}$ is a characteristic function of the $p$-adic disk with center in $i$ and radius $p^{-1}$. The operators defined by (3.2) and (3.3) satisfy the Cuntz algebra relations; thus, they give a representation of $O_{p}$.

Let us recall a few facts about $p$-adic and $q$-integrals. Let $p$ be a fixed odd prime and let $\mathbf{C}_{p}$ be the $p$-adic completion of the algebraic closure $\mathbf{Q}_{p}$. For $d$ a fixed positive integer with $(p, d)=1$, let $X=X_{d}=\lim _{\leftarrow} \mathbf{Z} / d p^{n}, X_{1}=\mathbf{Z}_{p}, X^{\star}=\bigcup_{(a, p)=1}^{0<a<d p} a+d p^{n} \mathbf{Z}_{p}$; then $a+d p^{n} \mathbf{Z}_{p}=\left\{x \in X \mid x \cong a \bmod d p^{n}\right\}, a \in \mathbf{Z}, 0<a<d p^{n}$. Suppose that $q \in \mathbf{C}$ and $|q|<1$. For any positive integer $N$, the measure $\mu_{q}$ is defined as

$$
\mu_{q}\left(a+d p^{N} \mathbf{Z}_{p}\right)=\frac{q^{a}}{d p^{N}}
$$

A typical $p$-adic interval is denoted by $I_{k}(a)=a+p^{k} \mathbf{Z}_{p}$.

Proposition 3.1. Given the $p$-adic representation of $O_{p}$ defined above, then there exists a measure $\mu$ such that

$$
\mu\left(I_{1}(i)\right)=\left\langle 1, S_{i} S_{i}^{\star} 1\right\rangle=\frac{q^{p}}{[p]_{q}} .
$$

Proof. By using the above $p$-adic representation of the Cuntz algebra with respect to the measure $\mu_{q}$ (see [12]),

$$
\begin{aligned}
\left\langle 1, S_{i} S_{i}^{\star} 1\right\rangle & =\frac{\sqrt{p}}{[p]_{q}} \int_{\|x-i\| \leq p^{-1}} 1\left(\frac{x-i}{p}\right) 1\left(i+p\left(\frac{x-i}{p}\right)\right) d \mu_{q}(x) \\
& =\frac{\sqrt{p}}{[p]_{q}} \int_{\|x-i\| \leq(p)^{-1}} 1\left(\frac{x-i}{p}\right) 1\left(i+p\left(\frac{x-i}{p}\right)\right) d \mu_{q}\left(\frac{x-i}{p}\right) \\
& =\frac{q^{p}}{[p]_{q}} .
\end{aligned}
$$

For a construction of induced measures, see [15, 16]. Let $A_{k}(a)$ be a $p$-adic system of partitions, where $a=\left(a_{1}, \ldots, a_{k}\right)$, and let $P_{k}(a)$ be the corresponding system of projections on $L^{2}\left(\mathbf{Z}_{p}, \mu_{q}\right)$.

Thus, the representation of the Cuntz algebra $O_{p}$ on $L^{2}\left(\mathbf{Z}_{p}, \mu_{q}\right)$ defined above gives rise to the following induced measure (which we denote by $\mu$ ) via a projection-valued measure:

$$
\mu\left(I_{1}(i)\right)=\left\langle 1, S_{i} S_{i}^{\star} 1\right\rangle=\frac{q^{p}}{[p]_{q}} .
$$

In [19], this measure has been shown to converge to a $q$-zeta function related to Bernoulli convolution measures.

\section{Abelian subalgebras and their Gelfand spaces}

The restrictions of representations of the Cuntz algebra to abelian subalgebras allow us to introduce associated Gelfand spaces. They will be needed in the next section, and are sketched below. 
Let $U=\{0, \ldots, p-1\}$ be an alphabet in $p$ letters.

We let $\Omega$ be the Gelfand space of the $C^{\star}$-subalgebra in $O_{p}$ generated by the elements $S_{k_{1}} \cdots S_{k_{m}} S_{k_{m}}^{\star} \cdots S_{k_{1}}^{\star}$ as the multi-indices $k=\left(k_{1}, \ldots, k_{m}\right)$ vary. Let $C(\Omega)$ be the canonical abelian $C^{\star}$-algebra inside $O_{p}$. We use the following lemma.

Lemma 4.1. Let $\Omega$ be the Gelfand space of the abelian $C^{\star}$-subalgebra $\mathcal{A}$ in $O_{p}$ generated by the monomials

$\left\{S_{k} S_{k}^{\star}: k\right.$ varying over all multi-indices formed from the alphabet $\left.U\right\}$.

Then, for every $i \in\{0, \ldots, p-1\}$, the mapping

$$
A \in \mathcal{A} \longmapsto S_{i} A S_{i}^{\star} \in \mathcal{A}
$$

induces an endomorphism $\tilde{\sigma}_{i}$ of $\Omega$.

Proof. This is an easy computation.

Remark. Specifically, the bijection between $\operatorname{End}(B(\mathcal{H})$ and $\operatorname{Rep}(\operatorname{Cuntz}, \mathcal{H})$ is up to the group of unitary (scalar) matrices acting on the generators for $O_{N}$; details below. By endomorphism in $B(\mathcal{H})$ we mean a normal $\star$-endomorphism sending the identity operator $I$ (that is, the unit in $B(\mathcal{H})$ to itself, $\sigma(I)=I$. The paper [3], and the papers cited there, contain details. More specifically, endomorphisms can be assigned a (Powers) index $N$ and, under the mentioned bijection, unital endomorphisms $\sigma$ in $B(\mathcal{H})$ of index $N$ correspond to representations of the Cuntz algebra $O_{N}$. We refer readers to these sources [3] for additional details. The idea is fundamental and dates back to Arveson. Full details are in [3]. In outline, it is as follows: if $\sigma$ is a given endomorphisms of $B(\mathcal{H})$ of Powers index $N$, then the relative commutant of $\sigma(B(\mathcal{H})$ ) in $B(\mathcal{H})$ is a type $I_{N}$ factor. By a careful identification of generators, one in turn then gets a representation of $O_{N}$. By this, we mean a system of $N$ operators $S_{i}$ in $\mathcal{H}$ satisfying the Cuntz relations. It can be shown that the representation of $O_{N}$ obtained in this way induces the given endomorphism $\sigma$. We have recalled the correspondence between endomorphism $\sigma$ and representation $\left(S_{i}\right)$ below.

If $\left(S_{i}\right)$ are isometries in a Hilbert space $\mathcal{H}$ generating a copy of the Cuntz $C^{\star}$-algebra, then

$$
\sigma(A):=\sum_{i} S_{i} A S_{i}^{\star}
$$

defines an endomorphism $\sigma$ of $B(\mathcal{H})$, the set of all the bounded operators on $\mathcal{H}$.

In fact, modulo an action by unitary matrices

$$
S_{i} \rightarrow \sum_{j} u_{j i} S_{j},
$$

this is a bijection between $\operatorname{End}(B(\mathcal{H}))$ and $\operatorname{Rep}(\mathrm{Cuntz}, \mathcal{H})$. The number of generators for the particular Cuntz algebra is called the Powers index of the endomorphism $\sigma$. 
The following result from [1] relates an induced measure to a specific Markov chain. This provides the motivation leading to the construction of Section 5 .

Lemma 4.2. Let $S_{i}$ be a representation of $O_{p}$ on $H$ arising from a multiresolution wavelet depending on a parameter $q$ see [1]. Let $a=\left(a_{1}, a_{2}, \ldots, a_{k}\right) \in \Gamma_{p}^{k}$ and $S_{a}:=$ $S_{\left(a_{1}\right)} \cdots S_{\left(a_{k}\right)}$ be such that $P_{k}(a)=S_{(a)} S_{(a)}^{*}$. Then

$$
\mu_{f}\left(I_{1}(a)\right)=\sum_{j \in \mathbf{Z}_{p}}\left|\left\langle H^{q} \psi_{a}(z), q^{j} t p, f\right\rangle\right|^{2}=\left([p]_{q}^{2}\right)^{-1} .
$$

Proof. See [1].

Assuming the unitarity condition on the filters $m_{j}$, we get

$$
\begin{aligned}
\mu_{f}\left(I_{k}(a)\right) & =\left\|E\left(I_{k}(a)\right) f\right\|^{2}=\left\|S_{(a)} S_{(a)^{*}} f\right\|^{2} \\
& =\left\langle f, S_{(a)} S_{(a)}^{*} f\right\rangle=\left\|S_{(a)}^{*} f\right\|^{2} \\
& =\sum\left|\left\langle e_{n}, S_{(a)}^{*} f\right\rangle\right|^{2}=\sum\left|\left\langle S_{(a)} e_{n}, f\right\rangle\right|^{2} .
\end{aligned}
$$

Using the Plancherel theorem for Hankel transforms for $a=a_{1}$, we get

$$
\mu_{f}\left(I_{1}(a)\right)=\sum_{j \in \mathbf{Z}_{p}}\left|\left\langle H^{q} \psi_{a}(z), q^{j} t p, f\right\rangle\right|^{2}=\left([p]_{q^{2}}\right)^{-1} .
$$

Let $\rho$ be an object in a tensor category that concretely we might take generated by composition of the canonical endomorphism $\sigma$. It was shown in [24] that there exists a Markov trace in quantum $S O_{p}$ such that the quantum dimension, denoted by $d_{q}^{\rho}$, is given by

$$
d_{q}^{\rho}=[p]_{q^{2}} .
$$

Therefore, it follows from Lemma 4.2 that the quantum dimension is the reciprocal of the induced measure:

$$
\mu_{f}\left(I_{k}(a)=\sum_{j \in \mathbf{Z}_{p}}\left|\left\langle H^{q} \psi_{a}(z), q^{j} t p, f\right\rangle\right|^{2}=d_{q}^{\rho},\right.
$$

where the representation of $O_{p}$ arises from a $q$-multiresolution wavelet [1]. This measure has a selfsimilarity behavior leading to the study of a Cuntz-Krieger type algebra of the next section.

\section{5. q-deformations of algebras and representations}

In this section, we combine the above tools and turn to a family of $q$-deformations and their representations, as introduced in Section 1. We extend the construction of the projection-valued measure to the case of a Cuntz-Krieger algebra with adjacency matrix depending on a parameter $q$. Thus, we continue the study of the family of measures from Lemma 4.2. In the context below, they will be associated with graph $C^{\star}$-algebras built on certain graphs: directed graphs with no sinks. 
A directed graph $G$ consists of a vertex set $V(G)$, an edge set $E(G)$, and range and source maps $r, s: E(G) \rightarrow V(G)$. Let $G$ be a finite directed graph with no sinks, that is, all vertices are sources. We choose the base vertex $v_{0} \in V(G)$ and we construct in the next proposition a measure

$$
\mu_{G, v_{0}}(v):=p_{e_{1}} \cdots p_{e_{k}},
$$

where $e_{1} \cdots e_{k}$ is the shortest path in the graph $G$ starting at $v_{0}$ and ending at $v$. Thus, we are considering a random walk on the graph $G$ starting at $v_{0}$, where at the first step we have the probability $p_{e}$ of moving to the nearby vertex $r(e)$ and probability 0 to remain at $v_{0}$. Our discussion of the measure construction, that is, the measure $\mu_{G}$ in the proposition below, is motivated by a longer project. Here we offer only a brief outline. The details of the project will be postponed to a future paper. Our aim is to prove the existence of Perron-Frobenius eigenvectors.

Outline of the construction: from the measure $\mu_{G}$, associated to a given semibranching function system, we will construct a matrix $A$ inducing in turn a CuntzKrieger relation. The range of the semibranching function system will then encode the matrix. Moreover, we work this in the reverse direction as well: from a given $q$-measure we aim to find the associated matrix. This will then give the existence of a Perron-Frobenius eigenvector.

Proposition 5.1. Let a finite directed graph having no sinks be given. Let $I_{e}$ be a $p$ adic interval as defined above. Then there exists a probability measure $\mu_{G}$ such that

$$
\mu_{G, v_{0}}(v):=p_{e_{1}} \cdots p_{e_{k}}
$$

where $p_{e_{i}}=\mu\left(I_{e_{i}}\right)$.

Proof. This follows as in [19], where we take $p_{e}=\mu\left(I_{e}\right)$, where $\mu$ is the measure from Proposition 3.1.

The basic idea in the construction of these, and related measures, is as follows: the generators for the Cuntz (or Cuntz-Krieger) $C^{\star}$-algebras are indexed by finite words, or by edges in a graph. In the latter case, we get projections in the $C^{\star}$-algebra indexed by vertices (in the same graph). Starting with a representation of the $C^{\star}$-algebra, in the technical step in our construction of the measures (projection-valued or scalarvalued), we are then making use of these projections. In every representation, we thus get consistency (see (5.6) and (5.5)), with the consistency thus implied by the underlying relations dictated by the particular Cuntz (or Cuntz-Krieger) algebra under consideration.

Implicit in this is a system of iterated subdivisions (represented symbolically by word systems and by shifts on finite words). This symbolic system is then used in turn to generate a $\sigma$-algebra of subsets on the real line. Summing up, we thus obtain the desired measure with properties dictated directly by the given representation. Referring to [19] for the details, this is the underlying idea behind our correspondence between representations of Cuntz (or Cuntz-Krieger) $C^{\star}$-algebras on the one hand, and associated measures on the other. 
Thus, the measure (5.2) gives the probability of reaching at time $k$ one of the vertices that are $k$ steps away from $v_{0}$.

Let $\sigma_{e}$ be a semibranching function system with the shift as coding map $\sigma$; then the measure $\mu$ applied to the interval $\sigma_{e^{\prime}} \circ I_{e}$ gives

$$
\mu\left(\sigma_{e^{\prime}} \circ I_{e}\right)=q^{e^{\prime}} \mu\left(I_{e}\right) .
$$

A Cuntz-Krieger family consists of mutually orthogonal projections $\left\{P_{v} \mid v \in V(G)\right\}$ and partial isometries $\left\{S_{e} \mid e \in E(G)\right\}$ satisfying

$$
P_{v}=\sum_{s(e)=v} S_{e} S_{e}^{\star}
$$

for all $v \in V(G)$, and

$$
P_{r(e)}=S_{e}^{\star} S_{e}
$$

for all edges $e \in E(G)$. The graph algebra $C^{\star}(G)$ is the universal $C^{\star}$-algebra generated by a Cuntz-Krieger $G$-family $S_{e}, P_{v}$.

We assume that the graph has no sinks, so that all vertices are sources. Then we have

$$
\sum_{v} S_{e} S_{e}^{\star}=1
$$

Let $\Lambda_{A}$ be the set of all admissible words in the alphabet $U$, where the admissibility condition is given by a $p \times p$ matrix $A$ (specified below) as

$$
\Lambda_{A}:=\left\{\omega=\left\{\omega_{n}\right\}_{n=0,1, \ldots} \mid \omega_{n} \in U, A_{\omega_{n}, \omega_{n+1}}=q^{\sigma_{n+1}(n)}\right\} .
$$

For $p=\operatorname{card}(E(G))$, we define the $p$ by $p$ matrix $A_{\omega_{i}, \omega_{i^{\prime}}}^{q}$ by

$$
A_{\omega_{i}, \omega_{i^{\prime}}}^{q}= \begin{cases}q^{\sigma_{\omega_{i^{\prime}}}\left(\omega_{i}\right)} & \text { if } r\left(\omega_{i}\right)=s\left(\omega_{i^{\prime}}\right), \\ 0 & \text { otherwise. }\end{cases}
$$

Then the relation $P_{r(e)}=S_{e}^{\star} S_{e}$, where now $e=\omega_{i}$, reads in an equivalent way as

$$
S_{e}^{\star} S_{e}=\sum_{e^{\prime}: r(e)=s\left(e^{\prime}\right)} A_{e e^{\prime}}^{q} S_{e^{\prime}} S_{e^{\prime}}^{\star},
$$

which gives the other Cuntz-Krieger type relation. See [29] for the particular case of a Cuntz-Krieger algebra.

We assume further that the matrix $A$ has the property that $A_{i, i}=1$ for every $i=$ $0, \ldots, p-1$, so that arbitrarily long strings made of the same letters are allowed as words in $\Lambda_{A}$. Proceeding as in [29], we topologize $\Lambda_{A}$ as a Cantor set by identifying it with a subset of $[0,1]$ of numbers whose $p$ expansion satisfies the admissibility condition. We remark that to be able to act with the shift on all of $\Lambda_{A}$, we identify the rational numbers in $[0,1]$ with infinite periodic sequences. We consider the Hilbert 
space to be $L^{2}\left(\Lambda_{A}, v_{A}\right)$, where $v_{A}$ is the Hausdorff measure in the Hausdorff dimension of $\Lambda_{A}$. Let $\sigma: \Lambda_{A} \rightarrow \Lambda_{A}, \sigma\left(x_{0} x_{1} \cdots x_{n} \cdots\right)=x_{1} x_{2} \cdots x_{n} \cdots$ be the self map, that is, the one-sided shift. For any $k \in \mathbf{N}$, we denote by $W_{k, A} \subset U^{k}$ the finite set of all the admissible words of length $k \in U$ :

$$
W_{k, A}=\left\{a=\left(a_{1}, \ldots, a_{k}\right) \in U^{k} \mid A_{a_{i}, a_{i+1}}=q^{\sigma_{i+1}(i)}\right\} .
$$

Let $\Lambda_{k, A}$ be the clopen subset of the Cantor set $\Lambda_{A}$ given by all the words that start with a given $a \in W_{k, A}$ :

$$
\Lambda_{k, A}(a)=\left\{\omega=\left(w_{1}, w_{2}, \ldots, w_{n}, \ldots\right) \in \Lambda_{A}:\left(w_{1}, \ldots, w_{k}\right)=a\right\} .
$$

In the next proposition, we show that there exists a partition and the corresponding operator-valued measure.

Proposition 5.2. The subset $\Lambda_{k, A}(a)$ as in (5.8) defines a p-adic system of projections $P_{k}(a)$ on the Hilbert space $H=L^{2}\left(\Lambda_{A}, v_{A}\right)$ and an orthonormal projection-valued measure $E$ on $B\left(\Lambda_{A}\right)$ such that

$$
E\left(\Lambda_{k, A}(a)\right)=P_{k}(a)
$$

Proof. For the case $q=1$, we have a Cuntz-Krieger algebra and the result follows from [29]. When $q \neq 1$, we need to observe the following: given the alphabet $U=\{0, \ldots, p-1\}$ for every $k \in \mathbf{N}$, the finite set of all admissible words in $U$ is such that

$$
W_{k, A}=\left\{a=\left(a_{1}, \ldots, a_{k}\right) \in U^{k} \mid A_{a_{i}, a_{i+1}}=q^{\sigma_{i+1}(i)}\right\} .
$$

We denote by $\Lambda_{k, A}(a)$ the clopen subset of the Cantor set $\Lambda_{A}, a \in W_{k, A}$,

$$
\Lambda_{k, A}(a)=\left\{w=\left(w_{1}, \ldots, w_{n-1}, \ldots,\right) \in \Lambda_{A} \mid\left(w_{1}, \ldots, w_{n}\right)=a\right\} .
$$

The subset $\Lambda_{k, A}$ defines a $p$-adic system of partitions for $\Lambda_{A}$. Then, as in [29], it follows that there exists a projection-valued measure on $B\left(\Lambda_{A}\right)$ such that

$$
E\left(\Lambda_{k, A}\right)=P_{k}(a)
$$

This concludes the proof.

In our case, we take

$$
P_{k}(a)=S_{a_{1}} \cdots S_{a_{k}} S_{a_{k}}^{\star} \cdots S_{a_{1}}^{\star} .
$$

Hence, we announce the following result.

THEOREM 5.3. The $C^{\star}$-algebra is generated by the relations

$$
\sum_{i} S_{i} S_{i}^{\star}=1
$$


and

$$
S_{e}^{\star} S_{e}=\sum_{e^{\prime}: r(e)=s\left(e^{\prime}\right)} S_{e^{\prime}} S_{e^{\prime}}^{\star}=\sum_{e^{\prime}} A_{e e^{\prime}}^{q} S_{e^{\prime}} S_{e^{\prime}}^{\star}
$$

for $q=1$ is a Cuntz-Krieger algebra. The Hausdorff measure $v_{A}$ on $\Lambda_{N, A^{q}}$ satisfies on the ranges of the semibranching function system $R_{i, q}$

$$
v\left(R_{i, q}\right)=p_{i}
$$

and the $p_{i}$ are the Perron-Frobenius eigenvectors of $A^{q}$ such that

$$
\sum_{j} A_{i, j}^{q} p_{j}=r\left(A^{q}\right) p_{i}
$$

with eigenvalue $r\left(A^{q}\right)$ the spectral radius.

\section{Conclusions}

As an example, we take the matrix $A$ given by the Markov chain as in [1]. Then the Cuntz-Krieger algebra with $A$ as adjacency matrix satisfies (5.4) and (5.7). Thus, the statement of Theorem 5.3 holds with the Perron-Frobenius eigenvector given by the induced measure from Section 3.

\section{Acknowledgements}

The second-named author (AMP) thanks the Max Planck Institute (Bonn) for support and hospitality. We further thank two anonymous referees for helpful suggestions, improving our presentation, and helping to clarify results.

\section{References}

[1] S. Albeverio, P. E. T. Jorgensen and A. M. Paolucci, 'Multiresolution wavelets analysis of integer scale Bessel functions', J. Math. Phys. 48 (2007), 073516.

[2] O. Bratteli and P. E. T. Jorgensen, 'Isometries, shifts, Cuntz algebras and multiresolution wavelet analysis of scale $N^{\prime}$, Integral Equations Operator Theory 28 (1997), 382-443.

[3] O. Bratteli and P. E. T. Jorgensen, 'Iterated function systems and permutation representations of the Cuntz algebra', Mem. Amer. Math. Soc. 139(663) (1999).

[4] O. Bratteli and P. E. T. Jorgensen, 'Wavelets through a looking glass', in: Applied and Numerical Harmonic Analysis (Birkhäuser, Boston, MA, 2002), p. xxii+398, ISBN:0-8176-4280-3.

[5] J. Cuntz, 'Simple $C^{*}$-algebras generated by isometries', Comm. Math. Phys. 56 (1977), 173-185.

[6] J. Cuntz, 'A class of $C^{\star}$-algebras and topological Markov chains', Invent. Math. 56 (1980), 251-268.

[7] I. Daubechies, Ten Lectures on Wavelets, CBMS-NSF Regional Conference Series in Applied Mathematics, 61 (Society for Industrial and Applied Mathematics, Philadelphia, PA, 1992).

[8] K. R. Davidson and D. R. Pitts, 'Invariant subspaces and hyper-reflexivity for free semigroup algebras', Proc. Lond. Math. Soc. 78 (1999), 401-430.

[9] D. E. Dutkay and P. E. T. Jorgensen, 'Harmonic analysis and dynamics for affine iterated function systems', Houston J. Math. 33(3) (2007), 877-905.

[10] D. E. Dutkay and P. E. T. Jorgensen, 'Martingales, endomorphisms, and covariant systems of operators in Hilbert space', J. Operator Theory 58(2) (2007), 269-310. 
[11] D. E. Dutkay and P. E. T. Jorgensen, A Duality Approach to Representations of Baumslag-Solitar Groups, Group Representations, Ergodic Theory, and Mathematical Physics: A Tribute to George W. Mackey, Contemporary Mathematics, 449 (American Mathematical Society, Providence, RI, 2008), pp. 99-127.

[12] G. Gasper and M. Rahman, Basic Hypergeometric Series, Encyclopedia of Mathematics and its Applications, 35 (Cambridge University Press, Cambridge, 1990).

[13] J. Glimm, 'Type I C*-algebras', Ann. of Math. (2) 73 (1961), 572-612.

[14] B. Jessen and A. Wintner, 'Distribution functions and the Riemann zeta function', Trans. Amer. Math. Soc. 38 (1935), 48-88.

[15] P. E. T. Jorgensen, 'Measures in wavelet decompositions', Adv. Appl. Math. 34 (2005), 561-590.

[16] P. E. T. Jorgensen, Analysis and Probability, Wavelets, Signals, Fractals, Graduate Texts in Mathematics, 234 (Springer, New York, 2006).

[17] P. E. T. Jorgensen, Certain Representations of the Cuntz Relations, and a Question on Wavelets Decompositions, Contemporary Mathematics, 414 (American Mathematical Society, Providence, RI, 2006), pp. 165-188.

[18] P. E. T. Jorgensen, Frame Analysis and Approximation in Reproducing Kernel Hilbert Spaces, Contemporary Mathematics, 451 (American Mathematical Society, Providence, RI, 2008), pp. 151-169.

[19] P. E. T. Jorgensen and A. M. Paolucci, 'Markov measures and extended zeta functions', submitted.

[20] P. E. T. Jorgensen and A. M. Paolucci, 'Wavelets in mathematical physics: $q$-oscillators', J. Phys. A: Math. Gen. 36 (2003), 6483-6494.

[21] P. E. T. Jorgensen, D. P. Proskurin and Y. S. Samoilenko, 'Generalized canonical commutation relations: representations and stability of universal enveloping $C^{*}$-algebra', in: Symmetry in Nonlinear Mathematical Physics, Pr. Inst. Mat. Nats. Akad. Nauk Ukr. Mat. Zastos., 2 (Nats̄ınal. Akad. Nauk Ukraïni Īnst. Mat., Kiev, 2002), pp. 456-460.

[22] P. E. T. Jorgensen, L. M. Schmitt and R. F. Werner, ' $q$-canonical commutation relations and stability of the Cuntz algebra', Pacific J. Math. 165 (1994), 131-151.

[23] P. E. T. Jorgensen and R. F. Werner, 'Coherent states of the $q$-canonical commutation relations', Comm. Math. Phys. 164 (1994), 455-471.

[24] E. C. Lance and A. M. Paolucci, 'Conjugation in braided $C^{\star}$-categories and orthogonal quantum groups', J. Math. Phys. 41(4) (2000), 2383-2394.

[25] F. Luef and Y. I. Manin, 'Quantum theta functions and Gabor frames for modulation spaces', Lett. Math. Phys. 88 (2009), 131-161.

[26] Y. I. Manin and M. Marcolli, Error-correcting codes and phase transitions', Math. Comput. Sci., to appear.

[27] M. Marcolli, Arithmetic Noncommutative Geometry, University Lecture Series, 36 (American Mathematical Society, Providence, RI, 2005).

[28] M. Marcolli, 'Modular curves, $C^{\star}$-algebras, and chaotic cosmology', in: Frontiers in Number Theory, Physics and Geometry, II (Springer, Berlin, 2006), pp. 361-372.

[29] M. Marcolli and A. M. Paolucci, 'Cuntz-Krieger algebras and wavelets on fractals', Complex Anal. Operator Theory, doi:10.1007/s11785-009-0044-y.

[30] I. Raeburn, Graph Algebras, CBMS Regional Conference Series in Mathematics, 103 (American Mathematical Society, Providence, RI, 2005).

\section{P. E. T. JORGENSEN, Department of Mathematics, University of Iowa, Iowa City, IA 52224, USA \\ e-mail: palle-jorgensen@uiowa.edu}

\section{A. M. PAOLUCCI, Max-Planck-Institut für Mathematik, Vivatsgasse 7, 53111 Bonn, Germany \\ e-mail: paolucci@mpim-bonn.mpg.de}

